\title{
ENDS OF MAPS AND APPLICATIONS
}

\author{
BY FRANK QUINN ${ }^{1}$
}

\begin{abstract}
Suppose $e: M \longrightarrow X$ is a map from a finite dimensional manifold to a locally compact space. Homotopy conditions on $e$ are developed under which it can be extended to a proper map $e^{\prime}: M^{\prime} \longrightarrow X$ by adding boundary to $M$. This is applied in several settings to obtain geometric conclusions from homotopy data. For example: an embedding $X \subset M$ of a manifold factor in a manifold of codimension $\geqslant 3$ is locally flat if and only if the complement $M-X$ is locally 1 connected at points of $X$.
\end{abstract}

A completion of a map $e: M \rightarrow X$ is a manifold $M^{\prime} \supseteq M$, with $M^{\prime}-M \subseteq$ $\partial M^{\prime}$, and an extension $e^{\prime}: M^{\prime} \rightarrow M$ which is proper. A map is proper if preimages of compact sets are compact.

MAIN TheOREm (THE END TheOREM [11]). Suppose $X$ is a locally compact locally 1-connected metric space. If $M$ is a manifold of dimension $\geqslant 6$, $e: M \rightarrow X$ is a map which is proper on $\partial M$, and the end of $e$ is onto, tame, and 1-LC, then e has a completion.

We define the terms used in the theorem. A neighborhood of the end is an open set $U \subset M$ such that $e \mid M-U$ is proper. The end of $e$ is onto if $e(U)=$ $X$ for every neighborhood $U$ of the end. The end of $e$ is $1-L C$ if for every $x \in$ $X$ and neighborhoods $U \subset M$ of the end, and $V \subset X$ of $x$, there are neighborhoods $U^{\prime} \subset U, V^{\prime} \subset V$ such that points (loops) in $U^{\prime} \cap e^{-1}\left(V^{\prime}\right)$ can be joined by by arcs (resp. are nullhomotopic) in $U \cap e^{-1}(V)$.

The end of $e$ is tame if there are homotopies pulling $M$ back from the end which are small in $X$. More precisely, for every $\epsilon>0$ and neighborhood $U$ of the end there is a neighborhood of the end $V \subset U$ and a homotopy $H: M \times I$ $\rightarrow M$ such that $H_{0}=I_{M}, H_{1}(M) \subset M-V, H_{t}(M-U) \subset M-V$, and for each $m \in M$ the diameter of the $\operatorname{arc} e H:\{m\} \times I \rightarrow X$ is less than $\epsilon$. Tameness is a necessary condition for a completion, since such homotopies can be obtained by pushing $M^{\prime}$ in a collar of $M^{\prime}-M \subseteq \partial M^{\prime}$.

If $X$ is a point, this theorem is exactly the theorem of Browder, Livesay, and Levine [1], later extended to nonsimply connected ends by Siebenmann [12].

The proof in outline is similar to the classical $X=$ pt. case. Homotopy and

Received by the editors August 9, 1978.

AMS (MOS) subject classifications (1970). Primary 57A99, 54C55, 57B05.

${ }^{1}$ Partially supported by the National Science Foundation. 
handlebody theory are used to show that there are highly connected manifold neighborhoods of the end, where $\epsilon$ control in $X$ is required in the connectivity. Once $n-3$ connected neighborhoods have been constructed, a "projective class group" obstruction is encountered. This formulated in terms of the geometric groups of Connell and Hollingsworth [5], and the proof is completed by essentially proving their conjectures.

We remark that there are useful relative and approximate versions of the end theorem, and an $\epsilon$ analog of the $h$-cobordism theorem over $X$. See [11] for details.

The end theorem and related techniques seem to be very useful in studying the more geometric aspects of the topology of manifolds. Roughly speaking, they replace many engulfing arguments.

Corollary 1. A 1-LC embedding of an ANR is a manifold of dimension $\geqslant 6$ has a mapping cylinder neighborhood.

The best previous result is that of Edwards [7], which applies to $X \subset M$ with $2 \operatorname{dim} X+3 \leqslant \operatorname{dim} M$.

The next result uses the theorem of Edwards [8] that a CE manifold image with the 2-disc property is a manifold.

Corollary 2. If $\operatorname{dim} X \geqslant 5$, the following are equivalent:

(a) $X$ is a $C E$ manifold image,

(b) $X$ is a manifold factor,

(c) $X \times \mathbf{R}^{2}$ is a manifold.

The best previous result is that of Cannon, Bryant, and Lacher [4], which requires the nonmanifold set be contained in a subset of dimension $\leqslant 1 / 2 \operatorname{dim} X$ -2 .

Corollary 3. An approximate fibration with total space a manifold, base a polyhedron, and fiber simply connected of dimension $\geqslant 5$, is concordant to a topological block bundle with manifold fiber.

Some results in this direction in very low dimensions have been obtained by Coram and Duvall [6], and by Goad [10].

CoRollary 4. If $M$ is a manifold of dimension $\geqslant 6$, and $X \subseteq M$ is codimension $\geqslant 3$, then $X$ is locally flat if and only if $X$ is a manifold factor and the embedding is 1-LC.

If $X$ is a manifold this result is due essentially to Bryant and Seebeck [2].

CoRollary 5. If $M$ is a homology manifold of dimension $\geqslant 6$ and $M-$ $\partial M$ is a manifold, then $\partial M \times \mathbf{R}^{2}$ is a manifold. Further, $\partial M$ is collared in $M$ if and only if $\partial M \times \mathbf{R}$ is a manifold and $\partial M \subset M$ is $1-L C$. 
Parts of this are due to Cernavskii, and Seebeck (see Ferry [9]), and to Cannon [3].

COROLlary 6. If $h: X \rightarrow M$ is a topological embedding of a manifold factor in a manifold of codimension $\geqslant 3$ and $\epsilon>0$, then $h$ can be $\epsilon$ approximated by a locally flat embedding.

This result uses an "uncrumpling" theorem of Cannon, Bryant, and Lacher [4]. For more detailed references and statements the reader should refer to the full version [11].

\section{REFERENCES}

1. W. Browder, J. Levine, and G. R. Livesay, Finding a boundary for an open mantfold, Amer. J. Math. 87 (1965), 1017-1028. MR 32 \#6473.

2. J. L. Bryant and C. L. Seebeck, III, Locally nice embeddings in codimension three, Quart. J. Math. Oxford Ser. (2) 21 (1970), 265-272. MR 44 \#7560.

3. J. W. Cannon, Taming codimension-one generalized submanifolds of $S^{n}$, Topology 16 (1977), 323-334.

4. J. W. Cannon, J. L. Bryant, and R. C. Lacher, The structure of generalized manifolds having nonmanifold set of trivial dimension, 1978 (preprint)

5. E. H. Connell and John Hollingsworth, Geometric groups and Whitehead torsion, Trans. Amer. Math. Soc. 140 (1969), 161-181.

6. D. S. Coram and P. F. Duvall, Approximate fibrations, Rocky Mountain J. Math. 7 (1977), 275-288.

7. R. D. Edwards, Siebenmann's variation of West's proof of the ANR theorem (to appear). print)

8. - Approximating certain cell-like maps by homeomorphisms, 1977. (pre-

9. Steve Ferry, Homotoping e-maps to homeomorphisms, Institute for Advanced Study, 1977 (preprint).

10. R. E. Goad, Local homotopy properties of maps, and approximation by fiber bundle projections, Thesis, Univ. of Georgia, 1976.

11. F. Quinn, Ends of maps, and applications, Virginia Polytechnic Institute and State University, 1978 (preprint).

12. L. C. Siebenmann, Obstructions to finding a boundary for an open manifold, Thesis, Princeton Univ., 1965.

DEPARTMENT OF MATHEMATICS, VIRGINIA POLYTECHNIC INSTITUTE AND STATE UNIVERSITY, BLACKSBURG, VIRGINIA 24061 